\title{
Oropharyngeal dysphagia: when swallowing disorders meet respiratory diseases
}

\author{
Eric Verin ${ }^{1,2,3,4}$, Pere Clavé ${ }^{4,5,6}$, Maria Rosaria Bonsignore ${ }^{7}$, Jean Paul Marie ${ }^{1,8}$, \\ Chloe Bertolus ${ }^{8,9}$, Thomas Similowski ${ }^{10,11}$ and Pierantonio Laveneziana 9,11
}

Affiliations: ${ }^{1}$ Normandie Univ, UNIROUEN, Rouen, France, and Institute for Research and Innovation in Biomedicine (IRIB), Rouen, France. ${ }^{2}$ Pôle 3R, Rouen University Hospital, Rouen, France. ${ }^{3}$ CRMPR les Herbiers, Bois Guillaume, France. ${ }^{4}$ European Society for Swallowing Disorders, Canet de Mar, Spain. ${ }^{5}$ Unitat d' Exploracions Funcionals Digestives, Department of Surgery, Hospital de Mataró, Universitat Autònoma de Barcelona, Mataró, Spain. ${ }^{6}$ Centro de Investigación Biomédica en Red de enfermedades hepáticas y digestivas (CIBERehd), Instituto de Salud Carlos III, Barcelona, Spain. ${ }^{7}$ DiBiMIS, University of Palermo, Italy, and Institute of Biomedicine and Molecular Immunology (IBIM), CNR, Palermo, Italy. ${ }^{8}$ Service de chirurgie cervico faciale, CHU de Rouen, Rouen, France. ${ }^{9}$ Assistance Publique-Hôpitaux de Paris (AP-HP), Groupe Hospitalier Pitié-Salpêtrière Charles Foix, Service de Chirurgie Maxilo faciale (Département "R3S"), Paris, France. ${ }^{10}$ Sorbonne Universités, UPMC Univ Paris 06, INSERM, UMRS1158 Neurophysiologie respiratoire expérimentale et clinique, Paris, France. ${ }^{11}$ Assistance Publique-Hôpitaux de Paris (AP-HP), Groupe Hospitalier Pitié-Salpêtrière Charles Foix, Service de Pneumologie et Réanimation Médicale (Département "R3S"), Paris, France.

Correspondence: Pr Eric Verin, Pôle 3R, Rouen University Hospital, 1 rue de Germont, Rouen Cedex 76031, France. E-mail: eric.verinachu-rouen.fr

@ERSpublications

Patients with chronic respiratory diseases should be screened for oropharyngeal dysphagia to prevent complications http://ow.ly/JaXl309wdNG

Cite this article as: Verin E, Clavé P, Bonsignore MR, et al. Oropharyngeal dysphagia: when swallowing disorders meet respiratory diseases. Eur Respir J 2017; 49: 1602530 [https://doi.org/10.1183/ 13993003.02530-2016].

The recent European Respiratory Society (ERS) Annual Meeting in London (September, 2016) has seen the beginning of a collaboration between the ERS and the European Society for Swallowing Disorders (ESSD) based on the importance (incidence and prevalence) of often unrecognised swallowing disorders in respiratory diseases.

Oropharyngeal dysphagia includes penetration of food, drink or saliva into the larynx, aspiration into the trachea, and oropharyngeal residue. It is a major complaint among many patients with neurological diseases and among the elderly [1]. The prevalence of functional oropharyngeal dysphagia is very high: it affects more than $30 \%$ of patients who have had a cerebrovascular accident, $52 \%-82 \%$ patients with neurodegenerative diseases, more than $35 \%$ of patients with head and neck diseases and more than $60 \%$ of elderly institutionalised patients [2].

Swallowing is defined as "the function of clearing food and drink through the oropharynx, and oesophagus into the stomach at an appropriate rate and speed". Oropharyngeal dysphagia is a clinical symptom, defined by the difficulty to move the alimentary bolus from the mouth to the oesophagus. However, the term is often used, not fully appropriately, to mean a disorder or disease of swallow function. Many patients affected are unaware of their swallow dysfunction. Swallowing is one of the most complex stereotyped patterns of motor behaviour involving over 25 pairs of muscles in the mouth, pharynx, larynx and oesophagus. The process of swallowing can be divided into three consecutive phases:

Received: Dec 232016 | Accepted: Jan 052017

Conflict of interest: Disclosures can be found alongside this article at erj.ersjournals.com

Copyright @ERS 2017 
oral phase (voluntary phase), pharyngeal phase (involuntary phase) and oesophageal phase. Swallowing requires precise coordination with the process of ventilation since both these processes share the pharynx as a conduit [3]. The coordinating site is located in the brainstem [4] and receives sensory influences from, and is under the influence of, pharyngeal swallowing afferences $[5,6]$; it is also influenced by afferences linked to the respiratory centre [7].

In healthy adults, swallowing occurs during the expiratory phase of respiration [3]. Swallowing apnoea, an involuntary pause in ventilation during swallowing, usually lasts between 0.5 and $1.0 \mathrm{~s}$ and occurs during expiration. Changes in the ventilation pattern can compromise swallowing and also swallowing-ventilation coordination [5, 8] because of interactions between the two central pattern generators [9]. In fact, modifying the ventilatory drive by inducing hypercapnia or by changing the respiratory mechanical or flow-resistive load, increases swallowing frequency and laryngeal irritation $[10,11]$. Concomitant oropharyngeal dysphagia in patients with chronic respiratory diseases [12] can increase exacerbations. Almost all of the studies on this subject showed a relationship between patients having oropharyngeal dysphagia and concomitant chronic obstructive pulmonary disease (COPD) [13, 14]. As a result, patients are more likely to suffer a rapid deterioration of their ventilatory function, aspiration and respiratory infection, and to be admitted more frequently to hospital. Given the direct consequences of oropharyngeal dysphagia, it is critical to determine whether patients with chronic respiratory diseases are at risk of dysphagia.

In respiratory diseases, $15 \%-20 \%$ of patients with COPD reported experiencing difficulties in swallowing [14]. Of particular note, dysphagia has been found to be a major complication of the surgical and radiotherapeutic treatment of oropharyngeal cancers [15]. Through the consequent lower respiratory tract infections, dysphagia can compromise the prognosis of patients otherwise cured of cancer, and alter their quality of life [16]

Oropharyngeal dysphagia may give rise to clinically relevant complications such as aspiration pneumonia, malnutrition and/or dehydration [17]. When a decrease in deglutition safety occurs, tracheobronchial aspiration results in pneumonia in $50 \%$ of cases [18], with an associated mortality of up to 50\% [19]. Impaired safety also limits the ability of patients to ingest all the calories and water that they need to be adequately nourished and hydrated [18]. Defining aspiration pneumonia as pneumonia occurring in patients with swallowing disorders, the Japanese Study Group on Aspiration Pulmonary Disease reported that the proportion of admissions due to aspiration pneumonia among all admissions due to pneumonia increases gradually with age, from $0 \%$ in those aged 50 years or younger to as high as $90 \%$ in those aged 90 years or older [20]. In a study by JokInEN et al. [21], the proportion of elderly patients with aspiration pneumonia increased to $93.8 \%$, while the proportion of elderly patients with other types of pneumonia decreased. A recent resolution of the Council of Europe claimed that undernutrition among hospital patients is highly prevalent and leads to extended hospital stays, prolonged rehabilitation, and diminished quality of life, and identified oropharyngeal dysphagia as a major contributor to malnutrition [22]. A recent study in a cohort of older patients admitted with an acute disease to a general hospital clearly revealed that patients with dysphagia and patients with malnutrition presented increased intra-hospital 6-month and 1-year mortality rates. The poorest outcome was for patients with both conditions (1-year mortality was 65.8\%) [18]. Oropharyngeal dysphagia has been identified as a serious risk factor for developing aspiration pneumonia in frail older people [23]. The pathogenesis of aspiration pneumonia in immunocompetent elderly people has been attributed to oropharyngeal colonisation of respiratory pathogens and subsequent aspiration-inhalation of infectious particles [24]. Oropharyngeal dysphagia has also been proposed as an independent risk factor associated with community-acquired pneumonia in the elderly. Moreover, the pathogenic mechanism that leads to oropharyngeal dysphagia in the frail elderly and in neurological patients constitutes a form of delayed airway protection [25]. Preserved swallowing and cough functions are thus essential in preventing deep aspirations and consequent chest infections. If both responses fail, silent aspiration (an aspiration without cough) will occur, a dangerous event because of the difficulties in detecting it clinically and its severe consequences [26].

The current state-of-the-art of oropharyngeal management is aimed at early identification of patients at risk for aspiration, assessment of alterations in the biomechanical events of the oropharyngeal swallow response, and prevention and treatment of the potential complications of dysphagia - such as aspiration pneumonia and malnutrition - by promoting posture and cough exercises [27, 28]. Treatment is also changing from compensatory strategies to the promotion of brain plasticity, both to recover swallow function and to improve brain-related swallowing dysfunction [6].

Videofluoroscopy (VFS) is the gold standard method for studying the oral and pharyngeal mechanisms of dysphagia and for evaluating the efficacy and safety of the swallow function [29]. VFS can identify the main signs of oropharyngeal dysfunction, which comprise delay in pharyngeal swallow, penetration of bolus into the laryngeal vestibule, tracheobronchial aspiration and oropharyngeal residue, and can assess the short-term effect of therapeutic strategies on dysphagic patients [30]. Using this technique, a study by 
CLAVÉ et al. [31] revealed that patients with neurogenic dysphagia presented high prevalence of impaired safety during liquid boluses, and that increasing bolus viscosity to nectar and pudding viscosity exerted a strong therapeutic effect on safety of deglutition. In contrast, increasing bolus volume was found to impair safety of deglutition in these patients.

Flexible endoscopic evaluation of swallowing (FEES) is also routinely used to assess aspiration. Furthermore, the sensitivity of FEES is comparable to that of videofluoroscopic swallowing examination in detecting aspiration [32]. FEES is an objective technique that permits pre-swallow assessment of both pharyngeal anatomy and physiology and the presence of pooled secretions in the pharynx, larynx or trachea [32]. It can be performed for as long as necessary to note fatigue with eating because there is no irradiation exposure, it provides pre- and post-swallowing visual biofeedback to the patient and family, and it allows for immediate assessment of dietary modifications [33].

The high prevalence of dysphagia among vulnerable patients and the dynamic condition of this symptom according to the natural history of each disease make it unfeasible to perform a videofluoroscopy or FEES on every patient or to repeat studies during disease evolution. Clinical screening assessment methods with high diagnostic accuracy are, therefore, needed to recognise patients with oropharyngeal dysphagia, to identify patients at risk of aspiration and who should be referred for a videofluoroscopy, and to help select the most appropriate bolus volume and viscosity for those patients who cannot easily undergo videofluoroscopy. To assess the diagnostic accuracy of a clinical bedside test, the ESSD developed the volume viscosity swallow test (V-VST), which is able to predict signs of dysphagia and impaired safety of deglutition (penetration, aspiration) observed during VFS studies, and to identify patients whose deglutition could be improved by increasing bolus viscosity [34].

To conclude, oropharyngeal dysphagia is highly prevalent among patients with chronic respiratory diseases, and also causes respiratory complications, mainly respiratory infections and pneumonia, showing the strong relationship between these conditions and its major complication - aspiration pneumonia. We recommend a policy of systematic and universal screening and assessment of oropharyngeal dysphagia among patients with these respiratory diseases to prevent the severe complications of their swallowing dysfunction. It concerns the elderly population, and patients with neurological disorders or head and neck cancer, but also patients with respiratory diseases. A collaboration between the two societies (ERS and ESSD) should help to develop such a programme for our patients.

\section{References}

$1 \quad$ Marik PE, Kaplan D. Aspiration pneumonia and dysphagia in the elderly. Chest 2003; 124: 328-336.

2 Kawashima K, Motohashi Y, Fujishima I. Prevalence of dysphagia among community-dwelling elderly individuals as estimated using a questionnaire for dysphagia screening. Dysphagia 2004; 19: 266-271.

3 Martin-Harris B, Brodsky MB, Price CC, et al. Temporal coordination of pharyngeal and laryngeal dynamics with breathing during swallowing: single liquid swallows. J Appl Physiol 2003; 94: 1735-1743.

4 Jean A. Brain stem control of swallowing: neuronal network and cellular mechanisms. Physiol Rev 2001; 81: 929-969.

5 Ouahchy Y, Messager V, Bon-Mardion N, et al. Laryngeal implications in swallowing-ventilation coordination. Eur Respir J 2011; 38: Suppl. 55, A4404.

6 Cabib C, Ortega O, Kumru H, et al. Neurorehabilitation strategies for poststroke oropharyngeal dysphagia: from compensation to the recovery of swallowing function. Ann N Y Acad Sci 2016; 1380: 121-138.

7 Ghannouchi I, Duclos C, Marie JP, et al. Modification in swallowing and ventilation co-ordination during hypercapnia, hypoxia, and tachypnea in unrestrained animals. Neurogastroenterol Motil 2013; 25: 308-e247.

8 Nishino T, Hasegawa R, Ide T, et al. Hypercapnia enhances the development of coughing during continuous infusion of water into the pharynx. Am J Respir Crit Care Med 1998; 157: 815-821.

9 Bautista TG, Sun QJ, Pilowsky PM. The generation of pharyngeal phase of swallow and its coordination with breathing: interaction between the swallow and respiratory central pattern generators. Prog Brain Res 2014; 212: 253-275.

10 Kijima M, Isono S, Nishino T. Coordination of swallowing and phases of respiration during added respiratory loads in awake subjects. Am J Respir Crit Care Med 1999; 159: 1898-1902.

11 Ghannouchi I, Speyer R, Doma K, et al. Swallowing function and chronic respiratory diseases: Systematic review. Respir Med 2016; 117: 54-64.

12 Chaves Rde D, Carvalho CR, Cukier A, et al. Symptoms of dysphagia in patients with COPD. J Bras Pneumol 2011; 37: 176-183.

13 Terada K, Muro S, Ohara T, et al. Abnormal swallowing reflex and COPD exacerbations. Chest 2010; 137: 326-332.

14 Mokhlesi B, Logemann JA, Rademaker AW, et al. Oropharyngeal deglutition in stable COPD. Chest 2002; 121: 361-369.

15 Lazarus C, Logemann JA, Pauloski BR, et al. Effects of radiotherapy with or without chemotherapy on tongue strength and swallowing in patients with oral cancer. Head Neck 2007; 29: 632-637.

16 Guibert M, Lepage B, Woisard V, et al. Quality of life in patients treated for advanced hypopharyngeal or laryngeal cancer. Eur Ann Otorhinolaryngol Head Neck Dis 2011; 128: 218-223.

17 Connolly MJ. Of proverbs and prevention: aspiration and its consequences in older patients. Age Ageing 2010; 39 : 2-4.

18 Carrión S, Cabré M, Monteis R, et al. Oropharyngeal dysphagia is a prevalent risk factor for malnutrition in a cohort of older patients admitted with an acute disease to a general hospital. Clin Nutr 2015; 34: 436-442. 
19 Cabré M, Serra-Prat M, Palomera E, et al. Prevalence and prognostic implications of dysphagia in elderly patients with pneumonia. Age Ageing 2010; 39: 39-45.

20 Teramoto S, Fukuchi Y, Sasaki H, et al. High incidence of aspiration pneumonia in community- and hospital-acquired pneumonia in hospitalized patients: a multicenter, prospective study in Japan. J Am Geriatr Soc 2008; 56: 577-579.

21 Jokinen $\mathrm{C}$, Heiskanen L, Juvonen $\mathrm{H}$, et al. Incidence of community-acquired pneumonia in the population of four municipalities in eastern Finland. Am J Epidemiol 1993; 137: 977-988.

22 Baine WB, Yu W, Summe JP. Epidemiologic trends in the hospitalization of elderly Medicare patients for pneumonia, 1991-1998. Am J Public Health 2001; 91: 1121-1123.

23 Cabré M, Serra-Prat M, Force L, et al. Oropharyngeal dysphagia is a risk factor for readmission for pneumonia in the very elderly persons: observational prospective study. J Gerontol A Biol Sci Med Sci 2014; 69: 330-337.

24 Rofes L, Arreola V, Romea M, et al. Pathophysiology of oropharyngeal dysphagia in the frail elderly. Neurogastroenterol Motil 2010; 22: 851-858, e230.

25 Almirall J, Bolíbar I, Serra-Prat M, et al. Relationship between the use of inhaled steroids for chronic respiratory diseases and early outcomes in community-acquired pneumonia. PLoS One 2013; 8: e73271.

26 Vilardell N, Rofes L, Nascimento WV, et al. Cough reflex attenuation and swallowing dysfunction in sub-acute post-stroke patients: prevalence, risk factors, and clinical outcome. Neurogastroenterol Motil 2017; 29: e12910.

27 Pitts T, Bolser D, Rosenbek J, et al. Impact of expiratory muscle strength training on voluntary cough and swallow function in Parkinson disease. Chest 2009; 135: 1301-1308.

28 Gourcerol G, Marie JP, Verin E. Glottis constriction response in healthy subjects. Respir Physiol Neurobiol 2005; 148: 303-308.

29 Logemann JA, Rademaker AW, Pauloski BR, et al. Normal swallowing physiology as viewed by videofluoroscopy and videoendoscopy. Folia Phoniatr Logop 1998; 50: 311-319.

30 Logemann JA. Oropharyngeal dysphagia and nutritional management. Curr Opin Clin Nutr Metab Care 2007; 10: 611-614.

31 Clavé $\mathrm{P}$, de Kraa M, Arreola V, et al. The effect of bolus viscosity on swallowing function in neurogenic dysphagia. Aliment Pharmacol Ther 2006; 24: 1385-1394.

32 Lim SH, Lieu PK, Phua SY, et al. Accuracy of bedside clinical methods compared with fiberoptic endoscopic examination of swallowing (FEES) in determining the risk of aspiration in acute stroke patients. Dysphagia 2001; 16: $1-6$.

33 Leder SB, Acton LM, Lisitano HL, et al. Fiberoptic endoscopic evaluation of swallowing (FEES) with and without blue-dyed food. Dysphagia 2005; 20: 157-162.

34 Clavé P, Arreola V, Romea M, et al. Accuracy of the volume-viscosity swallow test for clinical screening of oropharyngeal dysphagia and aspiration. Clin Nutr 2008; 27: 806-815. 